\title{
Characterization of $\{-1,0,+1\}$ Valued Functions in Discrete Tomography under Sets of Four Directions
}

\author{
Sara Brunetti ${ }^{1}$, Paolo Dulio ${ }^{2}$, and Carla Peri ${ }^{3}$ \\ 1 Dipartimento di Scienze Matematiche e Informatiche, Università di Siena, \\ Pian dei Mantellini 44, 53100, Siena, Italy \\ sara.brunetti@unisi.it \\ 2 Dipartimento di Matematica "F. Brioschi", Politecnico di Milano, \\ Piazza Leonardo da Vinci 32, I-20133 Milano, Italy \\ paolo.dulio@polimi.it \\ 3 Università Cattolica S. C., \\ Via Emilia Parmense, 8429122 Piacenza, Italy \\ carla.peri@unicatt.it
}

\begin{abstract}
In this paper we use the algebraic approach to Discrete Tomography introduced by Hajdu and Tijdeman to study functions $f$ : $\mathbb{Z}^{2} \rightarrow\{-1,0,+1\}$ which have zero line sums along the lines corresponding to certain sets of four directions.
\end{abstract}

Keywords: Discrete Tomography, $X$-ray, Unique Reconstruction, Generating Function.

\section{Introduction}

The main problems of Discrete Tomography deal with the reconstruction and the uniqueness questions. The first problem consists in the retrieval of an unknown finite subset of points of the so called lattice set $\mathbb{Z}^{2}$, from the knowledge of its $X$-rays taken along a given set of directions. The second problem focuses on the unique determination of a lattice set by means of its $X$-rays taken along a given set $S$ of lattice directions. In both cases we have to do with lattice sets (see 3 . and [4] for an overview of the topics). Recently, Hajdu and Tijdeman introduced in [1] an algebraic approach for discrete tomographical problems which is based on generating functions and divisibility properties of polynomials. This reveals to be useful for studying both the above problems, and especially uniqueness. In this paper we follow this idea in order to analyze functions $f: \mathbb{Z}^{2} \rightarrow\{-1,0,+1\}$ which has zero line sums along the lines corresponding to certain sets of four directions. Our study begins from the following result in [2]: for any fixed rectangle $A$ in $\mathbb{Z}^{2}$ there exists a "valid" set $S$ of four directions (at least when $A$ is not too "small"), depending only on the size of $A$, such that any two subsets of $A$ can be distinguished by means of $X$-rays along the directions in $S$. The proof was given by constructing explicitly a valid set $S$ of four directions in any

I. Debled-Rennesson et al. (Eds.): DGCI 2011, LNCS 6607, pp. 394-405, 2011.

(C) Springer-Verlag Berlin Heidelberg 2011 
possible case. We focus on sets $S$ of four directions. We first prove a necessary condition for $S$ to be a set of directions of uniqueness (Theorem 2). Then, in Theorem 3 , we find an algebraic necessary condition for a $\{-1,0,+1\}$ valued function $f$, under sets of four lattice directions taken according to Theorem 2 . in order to have $|f| \leq 1$. We also provide algebraic conditions for constructing such functions. Finally we deduce a uniqueness result in Corollary 1 .

\section{Notations and Preliminaries}

For all notations we refer to [2. Here we just recall the notion of valid set of directions. Let $a, b \in \mathbb{Z}$ with $\operatorname{gcd}(a, b)=1$ and $a \geq 0$, with the further assumption that $b=1$ if $a=0$. We call $(a, b)$ a lattice direction. Let $A=\left\{(i, j) \in \mathbb{Z}^{2}: 0 \leq\right.$ $i<m, 0 \leq j<n\}$. If $f: A \rightarrow \mathbb{Z}$ is a function, then $|f|=\max _{(i, j) \in A}\{|f(i, j)|\}$. A set $S=\left\{\left(a_{k}, b_{k}\right)\right\}_{k=1}^{d}$ of $d$ directions is said to be valid for $A$, if $\sum_{k=1}^{d} a_{k}<m$, and $\sum_{k=1}^{d}\left|b_{k}\right|<n$. In [2] L. Hajdu proved the following

Theorem 1. Let $m$ and $n$ be integers with $m \geq n \geq 5$ and $m \neq 6$, and let $A=\left\{(i, j) \in \mathbb{Z}^{2}: 0 \leq i<m, 0 \leq j<n\right\}$. Put $d=5$ if $(m, n) \in$ $\{(8,6),(8,8),(10,6),(12,6)\}$, and $d=4$ otherwise. Then there exists a valid set $S$ for $A$ consisting of $d$ directions depending only on $n$ if $n \geq 15$, and on $m$ and $n$ otherwise, such that if the function $f: A \rightarrow \mathbb{Z}$ has zero line sums along the lines corresponding to the directions in $S$ and $|f| \leq 1$, then $f$ is identically zero.

It follows that, under the above assumptions, if two functions $f, g: A \rightarrow\{0,1\}$ are tomographically equivalent (that is is they have equal line sums along the lines corresponding to the directions in $S$ ), then $f=g$. When $n \leq 15$ analogous results hold, but depending also on $m$ and in some cases with $d=5$ directions. See [2] for details.

Let $(a, b)$ be a lattice direction, then

$$
f_{(a, b)}(x, y)= \begin{cases}x^{a} y^{b}-1, & \text { if } a>0, b>0 \\ x^{a}-y^{-b}, & \text { if } a>0, b<0 \\ x-1, & \text { if } a=1, b=0 \\ y-1, & \text { if } a=0, b=1\end{cases}
$$

For any set $S$ of valid lattice directions, we denote by $F_{S}(x, y)=\sum f(i, j) x^{i} y^{j}$ the polynomial associated to $S$ defined as follows (see [2, pag. 19]):

$$
F_{S}(x, y)=\prod_{(a, b) \in S} f_{(a, b)}(x, y) .
$$

For any function $f: A \rightarrow \mathbb{Z}$, its generating function is defined by

$$
G_{f}(x, y)=\sum_{(i, j) \in A} f(i, j) x^{i} y^{j} .
$$

If $f$ has zero line sums along the lines taken in the directions of $S$, then $F_{S}(x, y)$ divides $G_{f}(x, y)$ over $\mathbb{Z}([1$, Lemma 3.1]). 
Further, we say that a point $(i, j) \in A$ is a multiple positive point for $f$ (or $\left.G_{f}\right)$ if $f(i, j)>1$. Analogously, $(i, j) \in A$ is said to be a multiple negative point for $f$ if $f(i, j)<-1$.

\section{$3 \quad$ Necessary Conditions for Unique Reconstructions}

In view of the tomographic problem of recognizing an arbitrary set of points in $\mathbb{Z}^{2}$ by means of $X$-rays corresponding to a given set $S$ of directions, an easy general necessary condition is the following.

Lemma 1. Let $S$ be any valid set of lattice directions, and let $F_{S}(x, y)=$ $\sum f(i, j) x^{i} y^{j}$ be the polynomial associated to $S$. If $f(i, j) \in\{-1,0,1\}$, then there exist two tomographically equivalent lattice sets with respect to $S$.

Proof. Let $F_{1}, F_{2}$ be the sets of points $(i, j)$ such that $f(i, j)=-1$ and $f(i, j)=$ 1 , respectively. Then $F_{S}(x, y)$ has zero sums along the directions in $S$, so that $F_{1}, F_{2}$ are tomographically equivalent.

The following is a useful result proved by L. Hajdu [2, Lemma 3.2], related to the problem of unique reconstruction.

Lemma 2. Let $S=\left\{\left(a_{k}, b_{k}\right)\right\}_{k=1}^{d}$ be any valid set of lattice directions. Suppose that $F_{S}(x, y)=\sum f(i, j) x^{i} y^{j}$ has a coefficient $f(i, j)$ outside the set $\{-1,0,1\}$. Then there exist two disjoint subsets $S_{1}$ and $S_{2}$ of $S$, such that $\left|S_{1}\right|=\left|S_{2}\right|(\bmod$ 2) and

$$
\sum_{(a, b) \in S_{1}}(a, b)=\sum_{(a, b) \in S_{2}}(a, b)
$$

Consider now a set $S=\left\{u_{1}, u_{2}, u_{3}, u_{4}\right\}$ of four distinct lattice directions $u_{1}=$ $(a, p), u_{2}=(b, q), u_{3}=(c, r), u_{4}=(d, s)$. In this case, Lemma 3 in [2] can be improved.

Lemma 3. Let $S=\left\{u_{1}, u_{2}, u_{3}, u_{4}\right\}$, and $F_{S}(x, y)=\sum f(i, j) x^{i} y^{j}$ its associated polynomial. Then $F_{S}(x, y)$ has a coefficient $f(i, j)$ outside the set $\{-1,0,1\}$ if and only if there exist two disjoint subsets $S_{1}$ and $S_{2}$ of $S$, such that $\left|S_{1}\right|=\left|S_{2}\right|$ $(\bmod 2)$ and

$$
\sum_{(a, b) \in S_{1}}(a, b)=\sum_{(a, b) \in S_{2}}(a, b)
$$

Proof. If $F_{S}(x, y)$ has a coefficient $f(i, j) \notin\{-1,0,1\}$, then, by [2, Lemma 3] there exist two disjoint subsets $S_{1}$ and $S_{2}$ of $S$, such that $\left|S_{1}\right|=\left|S_{2}\right|(\bmod 2)$ and (2) holds.

Suppose now the contrary. Since $|S|=4$, two disjoint subsets $S_{1}$ and $S_{2}$ of $S$ can exist, such that $\left|S_{1}\right|=\left|S_{2}\right|(\bmod 2)$ and (2) holds, if and only if one direction is the sum of the remaining three, or the sum of one pair equals the sum of the other. Therefore, up to permute the order in the choice of the directions, we must consider the following two cases. 
$C A S E$ 1. $u_{4}=u_{1}+u_{2}+u_{3}$

$C A S E$ 2. $u_{4}=u_{1}+u_{2}-u_{3}$

Let's show that in both cases we can always find a coefficient $f(i, j)$ which does not belong to the set $\{-1,0,1\}$.

$C A S E$ 1. Let $u_{4}=u_{1}+u_{2}+u_{3}$ so that $(d, s)=(a+b+c, p+q+r)$. Since $S$ consists of distinct directions, without loss of generality we can assume

$$
0 \leq a \leq b \leq c, \quad b, c \neq 0
$$

Moreover, $F_{S}(x, y)$ is formed by monomials as in Table 1 (up to a common factor $y^{h}$ if $p, q, r$ are not all positive). Consequently the coefficient 2 vanishes if the monomial $x^{a+b+c} y^{p+q+r}$ is equal to one of the monomials with a positive coefficient. Note that

1. $x^{2(a+b+c)} y^{2(p+q+r)} \neq x^{a+b+c} y^{p+q+r}$, by (3).

2. If $x^{2 a+b+c} y^{2 p+q+r}=x^{a+b+c} y^{p+q+r}$, then $a=0$ and $p=0$, which is impossible for a direction $(a, p)$. For the same reason it is $x^{b+c} y^{q+r} \neq x^{a+b+c} y^{p+q+r}$

3. $x^{a+2 b+c} y^{p+2 q+r} \neq x^{a+b+c} y^{p+q+r}$ since $b \neq 0$. For the same reason it is $x^{a+c} y^{p+r} \neq x^{a+b+c} y^{p+q+r}$

4. $x^{a+b+2 c} y^{p+q+2 r} \neq x^{a+b+c} y^{p+q+r}$ since $c \neq 0$.

5. $x^{a+b} y^{p+q} \neq x^{a+b+c} y^{p+q+r}$ since $c \neq 0$.

6. $1 \neq x^{a+b+c} y^{p+q+r}$ since $a+b+c>0$.

Table 1. Monomials of $F_{S}(x, y)$ in CASE 1

\begin{tabular}{|cc|}
\hline sign + & sign - \\
$x^{2(a+b+c)} y^{2(p+q+r)}$ & $x^{2 a+2 b+c} y^{2 p+2 q+r}$ \\
$x^{2 a+b+c} y^{2 p+q+r}$ & $x^{2 a+b+2 c} y^{2 p+q+2 r}$ \\
$x^{a+2 b+c} y^{p+2 q+r}$ & $x^{a+2 b+2 c} y^{p+2 q+2 r}$ \\
$x^{a+b+2 c} y^{p+q+2 r}$ & $2 x^{a+b+c} y^{p+q+r}$ \\
$x^{a+b} y^{p+q}$ & $x^{a} y^{p}$ \\
$x^{a+c} y^{p+r}$ & $x^{b} y^{q}$ \\
$x^{b+c} y^{q+r}$ & $x^{c} y^{r}$ \\
1 & \\
\hline
\end{tabular}

This shows that in $C A S E 1$ there is a coefficient $f(i, j)$ which does not belong to the set $\{-1,0,1\}$.

$C A S E$ 2. Let $u_{4}=u_{1}+u_{2}-u_{3}$ so that $(d, s)=(a+b-c, p+q-r)$. Without loss of generality we can assume

$$
0 \leq a \leq b, \quad 0 \leq a+b-c, \quad b \neq 0
$$

Now $F_{S}(x, y)$ is formed by monomials as in Table 2 (still up to a common factor $y^{h}$ if $p, q, r$ are not all positive). 
Table 2. Monomials of $F_{S}(x, y)$ in CASE 2

\begin{tabular}{|cc|}
\hline sign + & sign - \\
$x^{2(a+b)} y^{2(p+q)}$ & $x^{2(a+b)-c} y^{2(p+q)-r}$ \\
$x^{2 a+b-c} y^{2 p+q-r}$ & $x^{2 a+b} y^{2 p+q}$ \\
$x^{a+2 b-c} y^{p+2 q-r}$ & $x^{a+2 b} y^{p+2 q}$ \\
$2 x^{a+b} y^{p+q}$ & $x^{a+b+c} y^{p+q+r}$ \\
$x^{a+c} y^{p+r}$ & $x^{a+b-c} y^{p+q-r}$ \\
$x^{b+c} y^{q+r}$ & $x^{a} y^{p}$ \\
1 & $x^{b} y^{q}$ \\
& $x^{c} y^{r}$ \\
\hline
\end{tabular}

The coefficient 2 vanishes if the monomial $x^{a+b} y^{p+q}$ equals one with negative sign. Note that

1. If $x^{2(a+b)-c} y^{2(p+q)-r}=x^{a+b} y^{p+q}$, then $c=a+b$ and $r=p+q$, deriving $d=0$ and $s=0$, which is impossible for a direction $(d, s)$. For the same reason it is $x^{c} y^{r} \neq x^{a+b} y^{p+q}$

2. If $x^{2 a+b} y^{2 p+q}=x^{a+b} y^{p+q}$, then $a=0$ and $p=0$, which is impossible for a direction $(a, p)$. For the same reason it is $x^{b} y^{q} \neq x^{a+b} y^{p+q}$

3. $x^{a+2 b} y^{p+2 q} \neq x^{a+b} y^{p+q}$, since $b \neq 0$ by (4).

4. If $x^{a+b+c} y^{p+q+r}=x^{a+b} y^{p+q}$, then $c=0$ and $r=0$, which is impossible for a direction $(c, r)$. Analogously we have $x^{a+b-c} y^{p+q-r} \neq x^{a+b} y^{p+q}$.

5. $x^{a} y^{p} \neq x^{a+b} y^{p+q}$, since $b \neq 0$ by (4).

Consequently, also in $C A S E 2$ there is a coefficient $f(i, j)$ which does not belong to the set $\{-1,0,1\}$, and the proof is complete.

From Lemma 3 we derive the following necessary condition of unique reconstruction.

Theorem 2. The sets of four directions that uniquely determine the lattice sets in a finite grid must be of the form $\left\{u_{1}, u_{2}, u_{3}, u_{1}+u_{2} \pm u_{3}\right\}$.

Proof. By Lemma 1 a set $S=\left\{u_{1}, u_{2}, u_{3}, u_{4}\right\}$ of four direction could be a set of uniqueness if the associated polynomial $F_{S}(x, y)=\sum f(i, j) x^{i} y^{j}$ has at least a coefficient $f(i, j) \notin\{-1,0,1\}$. By $C A S E 1$ and $C A S E 2$ in Lemma 3 this occurs if and only if $u_{4}=u_{1}+u_{2} \pm u_{3}$.

Remark 1. Let $S$ be a set of four lattice directions such that $F_{S}(x, y)$ has some coefficient outside $\{-1,0,1\}$. By Lemma 3 we know that there exist two disjoint subsets $S_{1}, S_{2}$ of $S$, such that $\left|S_{1}\right|=\left|S_{2}\right|(\bmod 2)$, and $\sum_{u \in S_{1}} u=\sum_{u \in S_{2}} u$, and by Theorem 2 we get that $S=S_{1} \cup S_{2}$, where $S_{1}=\left\{u_{1}, u_{2}, u_{3}\right\}, S_{2}=\left\{u_{4}\right\}$ in CASE 1 , and $S_{1}=\left\{u_{1}, u_{2}\right\}, S_{2}=\left\{u_{3}, u_{4}\right\}$ in CASE 2. For such a set $S$ we define the set $S_{1}-S_{2}$ as follows

$$
S_{1}-S_{2}=\left\{ \pm\left(u_{i}-u_{j}\right), u_{i} \in S_{1}, u_{j} \in S_{2}\right\}
$$




\section{Sets of Four Directions for Unique Reconstructions}

In this section we analyze the functions $g: \mathbb{Z}^{2} \rightarrow \mathbb{Z}$ which have zero line sums along the lines corresponding to a given set of directions $S,|g| \leq 1$, and vanish outside a finite latice set. Notice that these properties are invariant by integer translations, so that we can argue up to integer translations, when necessary.

Let $S=\left\{u_{1}, u_{2}, u_{3}, u_{4}\right\}$ be a valid set of four lattice directions, and let

$$
F_{S}(x, y)=\prod_{(a, b) \in S} f_{(a, b)}(x, y)=\sum f(i, j) x^{i} y^{j}
$$

be its associated polynomial. Define $Q(S)=\left\{(i, j) \in \mathbb{Z}^{2}: f(i, j) \neq 0\right\}$. We partition the set $Q(S)$ into two disjoint subsets, according to the sign of the weight $f(i, j)$ of the point $(i, j) \in Q(S)$ :

$$
\mathcal{P}=\{(i, j) \in Q(S): f(i, j)>0\} \quad \mathcal{N}=\{(i, j) \in Q(S): f(i, j)<0\}
$$

Notice that if $b \geq 0$ for all $(a, b) \in S$ then the set $\mathcal{P}$ consists of the points

$$
\begin{aligned}
& \mathbf{0}, \quad u_{1}+u_{2}, u_{1}+u_{3}, u_{1}+u_{4}, \\
& u_{2}+u_{3}, u_{2}+u_{4}, u_{3}+u_{4}, u_{1}+u_{2}+u_{3}+u_{4}
\end{aligned}
$$

and the set $\mathcal{N}$ consists of the points

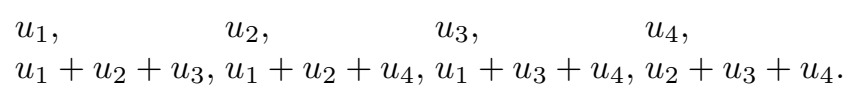

not all necessarily distinct. If $b<0$ for some $(a, b) \in S$, then the sets $\mathcal{P}$ and $\mathcal{N}$ consist of the points (6) and (7), respectively, translated by the vector $(0,-h)$, where $h$ is the sum of negative values of $b$ for $(a, b) \in S$. In the following we shall assume that $\mathcal{P}$ and $\mathcal{N}$ are represented by (6) and (7), respectively, since the properties we are looking for are invariant by integer translations. From the algebraic viewpoint, this corresponds with substituting the polynomial $F_{S}(x, y)$ by the rational function $y^{d} F_{S}(x, y)$.

For $u=(h, k) \in \mathbb{Z}^{2}$, let $f_{-}^{u}: \mathbb{Z}^{2} \rightarrow \mathbb{Z}, f_{+}^{u}: \mathbb{Z}^{2} \rightarrow \mathbb{Z}$ be the maps whose generating functions are $G_{f_{-}^{u}}(x, y)=\left(x^{h} y^{k}-1\right) F_{S}(x, y)$ and $G_{f_{+}^{u}}(x, y)=\left(x^{h} y^{k}+\right.$ 1) $F_{S}(x, y)$, respectively. Notice that for $h<0$ or $k<0, G_{f_{-}^{u}}, G_{f_{+}^{u}}$ are rational functions, which can be mapped to polynomials by integer translations.

Lemma 4. Let $S=\left\{u_{1}, u_{2}, u_{3}, u_{1}+u_{2} \pm u_{3}\right\}$ be a set of four lattice directions, and let $u \in \mathbb{Z}^{2}$. If $u \in S$ or $-u \in S$, then $\left|f_{-}^{u}\right|>1$.

Proof. If $u=(h, k) \in S$, then $u \in \mathcal{N}$ by (7), so that $f(h, k)<0$. By definition the coefficient of the monomial $x^{h} y^{k}$ in the generating function $G_{f_{-}^{u}}$ of $f_{-}^{u}$ is given by $f(0,0)-f(h, k)$. Moreover, since $(0,0) \in \mathcal{P}$ we have $f(0,0)-f(h, k) \geq 2$, so that $\left|f_{-}^{u}\right|>1$. If $-u \in S$, then $-u \in \mathcal{N}$ by (7), so that $f(-h,-k)<0$. By definition the coefficient of the monomial of degree zero in the generating function $G_{f_{-}^{u}}$ of $f_{-}^{u}$ is given by $f(-h,-k)-f(0,0) \leq-2$, so that $\left|f_{-}^{u}\right|>1$. 
Lemma 5. Let $S=\left\{u_{1}, u_{2}, u_{3}, u_{1}+u_{2} \pm u_{3}\right\}$ be a set of four lattice directions. For each $u_{i} \in S$ there exists $u=(h, k) \in\left(S_{1}-S_{2}\right)$ such that $u_{i}$ is a multiple negative point for $f_{+}^{u}$.

Proof. Let $u_{i}=\left(h_{i}, k_{i}\right) \in S$ and let $u=(h, k) \in\left(S_{1}-S_{2}\right)$ such that $u=u_{i}-u_{j}$ for some $u_{j}=\left(h_{j}, k_{j}\right)$. Then $\left(h_{i}, k_{i}\right)=\left(h+h_{j}, k+k_{j}\right)$ so that the coefficient of the monomial $x^{h_{i}} y^{k_{i}}$ in the generating function $G_{f_{+}^{u}}$ of $f_{+}^{u}$ is given by $f\left(h_{i}, k_{i}\right)+$ $f\left(h_{j}, k_{j}\right)$. Since $u_{i}, u_{j} \in \mathcal{N}$, then $f\left(h_{i}, k_{i}\right)+f\left(h_{j}, k_{j}\right) \leq-2$. Therefore, $u_{i}$ is a multiple negative point for $f_{+}^{u}$.

Lemma 6. Let $S=\left\{u_{1}, u_{2}, u_{3}, u_{4}\right\}$ be a set of four lattice directions, such that $u_{4}=u_{1}+u_{2} \pm u_{3}$. Then the following hold

1. $\left|f_{-}^{u}\right| \leq 1$ if and only if $u=0$, or $u \in\left(S_{1}-S_{2}\right)$ and $u_{4}=u_{1}+u_{2}+u_{3}$, or $u \in\left(S_{1}-S_{2}\right) \cup\left\{ \pm\left(u_{1}+u_{2}\right)\right\}$ and $u_{4}=u_{1}+u_{2}-u_{3}$.

2. $\left|f_{+}^{u}\right| \leq 1$ if and only if $u \in S$ or $-u \in S$.

Proof. We first note that if $u=0$ then $\left|f_{-}^{0}\right|$ is identically zero, so that $\left|f_{-}^{0}\right| \leq 1$, while $\left|f_{+}^{0}\right|=2 f$, so that $\left|f_{+}^{0}\right|>1$.

If $u \in S$ or $-u \in S$, then, by Lemma $4\left|f_{-}^{u}\right|>1$. Consequently, in order to get $\left|f_{-}^{u}\right| \leq 1$, we must choose $\pm u \notin S$. Analogously, if $u \in\left(S_{1}-S_{2}\right)$, then $u= \pm\left(u_{i}-u_{j}\right)$ for some $u_{i}, u_{j} \in S$. By Lemma 5, either $u_{i}$ or $u_{j}$ is a multiple negative point of $f_{+}^{u}$ so that $\left|f_{+}^{u}\right|>1$. Consequently, in order to get $\left|f_{+}^{u}\right| \leq 1$, we must choose $u \notin\left(S_{1}-S_{2}\right)$.

Consider the multiple (positive or negative) point $w=(1 / 2)\left(u_{1}+u_{2}+u_{3}\right.$ $\left.+u_{4}\right) \in Q(S)$ for $F_{S}$ (see Table1 and Table 2). Let $z \in \mathbb{Z}^{2}$ such that $z=w+u$. If $z \notin Q(S)$ then $f_{ \pm}^{u}(z)=f(w)$, so that $z$ is a multiple point (positive or negative) for $f_{ \pm}^{u}$, and consequently $\left|f_{ \pm}^{u}\right|>1$. Then, in order to get $\left|f_{ \pm}^{u}\right| \leq 1$, we need $z \in Q(S)$.

Let us first suppose that $u_{4}=u_{1}+u_{2}+u_{3}$, so that $w$ is a multiple negative point for $F_{S}$. Then, by (6) and (7), to have $z \in Q(S)$, the vector $u$ must be selected as follows

$$
\begin{aligned}
& u=\mathbf{0}, \quad u= \pm u_{1}, \quad u= \pm u_{2}, \quad u= \pm u_{3}, \\
& u= \pm u_{4}, u= \pm\left(u_{4}-u_{1}\right), u= \pm\left(u_{4}-u_{2}\right), u= \pm\left(u_{4}-u_{3}\right) \text {, }
\end{aligned}
$$

The case $u=\mathbf{0}$ has already been considered. By Lemma 4 the cases $\pm u \in S$ cannot give $\left|f_{-}^{u}\right| \leq 1$. To this, the only possible choice is $u \in\left(S_{1}-S_{2}\right)$. The explicit computations of $G_{f_{-}^{u}}(x, y)=\left(x^{h} y^{k}-1\right) F_{S}(x, y)$ for $(h, k) \in\left(S_{1}-S_{2}\right)$ show that in fact $\left|f_{-}^{u}\right| \leq 1$ in all the cases, and (11) is proved.

The cases $u \in\left\{ \pm\left(u_{4}-u_{1}\right), \pm\left(u_{4}-u_{2}\right), \pm\left(u_{4}-u_{3}\right)\right\}$ correspond to $u \in$ $\left(S_{1}-S_{2}\right)$, and consequently cannot give $\left|f_{+}^{u}\right| \leq 1$ by Lemma 5. To this, the only possible choices are $u \in S$ or $-u \in S$. The explicit computations of $G_{f_{+}^{u}}(x, y)=\left(x^{h} y^{k}+1\right) F_{S}(x, y)$ for such $h, k$ show that in fact $\left|f_{+}^{u}\right| \leq 1$ in all the cases, and (2) is proved. 
Now, assume $u_{4}=u_{1}+u_{2}-u_{3}$. Then, by (6) and (7), to have $z \in Q(S)$ the vector $u$ must be selected as follows

$$
\begin{aligned}
& u=0, \quad u= \pm u_{1}, \quad u= \pm u_{2}, \quad u= \pm u_{3} \\
& u= \pm u_{4}, u= \pm\left(u_{3}-u_{1}\right), u= \pm\left(u_{3}-u_{2}\right), u= \pm\left(u_{1}+u_{2}\right)
\end{aligned}
$$

The case $u=\mathbf{0}$ has already been considered above. If $u \in S$ or $-u \in S$ then $\left|f_{-}^{u}\right|>1$, by Lemma 4. Therefore, we remain with $u \in\left(S_{1}-S_{2}\right) \cup\left\{ \pm\left(u_{1}+u_{2}\right)\right\}$, and the explicit computations of $G_{f_{-}^{u}}(x, y)=\left(x^{h} y^{k}-1\right) F_{S}(x, y)$ for such $u$ show that it is always $\left|f_{-}^{u}\right| \leq 1$. Therefore, (1) is proved also in this case.

Differently, to get $\left|f_{+}^{u}\right| \leq 1$, we must avoid $u \in\left(S_{1}-S_{2}\right)$, by Lemma 5, and $u= \pm\left(u_{1}+u_{2}\right)$, as it is shown by the explicit computations of $G_{f_{+}^{u}}(x, y)=$ $\left(x^{h} y^{k}+1\right) F_{S}(x, y)$. Thus, we remain with $u \in S$ or $-u \in S$. The explicit computations of $G_{f_{+}^{u}}(x, y)=\left(x^{h} y^{k}+1\right) F_{S}(x, y)$ for such $u$ show that it is always $\left|f_{+}^{u}\right| \leq 1$. Therefore (2) is proved also in this case.

From the above lemmas, we can derive a necessary condition for a $\{-1,0,+1\}$ valued function $g$, which has zero line sums along the lines corresponding to sets of four lattice directions taken according to Theorem 2 , in order to have $|g| \leq 1$.

Theorem 3. Let $S=\left\{u_{1}, u_{2}, u_{3}, u_{1}+u_{2} \pm u_{3}\right\}$ be a set of four lattice directions. Let $g: \mathbb{Z}^{2} \rightarrow \mathbb{Z}$ be a non trivial function which has zero line sums along the lines corresponding to the directions in $S$, and vanishes outside a finite lattice set. If $|g| \leq 1$ then there exists $r \in \mathbb{N}$ such that

$$
G_{g}(x, y)=\sum_{t=1}^{r} \delta(t) x^{i(t)} y^{j(t)} F_{S}(x, y),
$$

where $\delta(t)= \pm 1$, and for each $t \in\{1, \ldots, r\}$, there exists $t^{\prime} \in\{1, \ldots, r\}$ such that the vector $u(t)=(i(t), j(t))-\left(i\left(t^{\prime}\right), j\left(t^{\prime}\right)\right)$ satisfies the following conditions

1. $u(t)=0$, or $u(t) \in\left(S_{1}-S_{2}\right)$ and $u_{4}=u_{1}+u_{2}+u_{3}$, or $u(t) \in\left(S_{1}-S_{2}\right) \cup$ $\left\{ \pm\left(u_{1}+u_{2}\right)\right\}$ and $u_{4}=u_{1}+u_{2}-u_{3}$, if $\delta(t) \neq \delta\left(t^{\prime}\right)$.

2. $u(t) \in S$ or $-u(t) \in S$ if $\delta(t)=\delta\left(t^{\prime}\right)$.

Proof. By Theorem 1 in [1], $G_{g}(x, y)=P(x, y) F_{S}(x, y)$ for some polynomial $P(x, y) \in \mathbb{Z}[x, y]$. Each monomial $a_{i j} x^{i} y^{j}$ of $P(x, y)$ can be split into a sum of monomials with coefficients \pm 1 , so that $P(x, y)=\sum_{t=1}^{r} \delta(t) x^{i(t)} y^{j(t)}$, and

$$
G_{g}(x, y)=\sum_{t=1}^{r} \delta(t) x^{i(t)} y^{j(t)} F_{S}(x, y),
$$

where $\delta(t)= \pm 1$. Consider the multiple (positive or negative) point

$$
w=(1 / 2)\left(u_{1}+u_{2}+u_{3}+u_{4}\right)
$$

for $F_{S}$. We have $|f(w)|=2$, where $f$ is the function with generating function $F_{S}$. By multiplying $F_{S}(x, y)$ times $x^{i(t)} y^{j(t)}$, the value $f(w)=2$ of the 
the multiple (positive or negative) point $w$ is attached to the translated point $w+(i(t), j(t))$. Since $|g| \leq 1$, such value must be reduced by adding to the corresponding monomial a monomial with same degree and coefficient with opposite sign. Thus, for each $t \in\{1, \ldots, r\}$, some point $z_{t} \in Q(S)$ must exist, and some monomial $\delta\left(t^{\prime}\right) x^{i\left(t^{\prime}\right)} y^{j\left(t^{\prime}\right)} \neq \delta(t) x^{i(t)} y^{j(t)}$ of $P(x, y)$, such that $z_{t}+\left(i\left(t^{\prime}\right), j\left(t^{\prime}\right)\right)=w+(i(t), j(t))$. It follows that the function

$$
\begin{aligned}
& \left(\delta(t) x^{i(t)} y^{j(t)}+\delta\left(t^{\prime}\right) x^{i\left(t^{\prime}\right)} y^{j\left(t^{\prime}\right)}\right) F_{S}(x, y)= \\
& x^{i\left(t^{\prime}\right)} y^{j\left(t^{\prime}\right)}\left(\delta(t) x^{\left.i(t)-i\left(t^{\prime}\right)\right)} y^{j(t)-j\left(t^{\prime}\right)}+\delta\left(t^{\prime}\right)\right) F_{S}(x, y)
\end{aligned}
$$

has no multiple points. Let us define $u(t)=(i(t), j(t))-\left(i\left(t^{\prime}\right), j\left(t^{\prime}\right)\right)=z_{t}-w$. By Lemma 6 applied to the vector $u(t)$ and to the function

$$
\left(\delta(t) x^{\left.i(t)-i\left(t^{\prime}\right)\right)} y^{j(t)-j\left(t^{\prime}\right)}+\delta\left(t^{\prime}\right)\right) F_{S}(x, y),
$$

we get either (1) or (2), depending on the signs of $\delta(t)$ and $\delta\left(t^{\prime}\right)$, which provide a necessary condition for $|g| \leq 1$.

We now provide some algebraic conditions which show how a $\{-1,0,+1\}$ valued function $g$, with required norm $|g| \leq 1$, can be constructed. We first fix some notations. Let $g: \mathbb{Z}^{2} \rightarrow \mathbb{Z}$ be a non trivial function whose generating function is defined by $G_{g}(x, y)=P(x, y) F_{S}(x, y)$, for some polynomial $P(x, y)$, consisting of $r$ monomials. For $h \in\{1, \ldots, r\}$, let $g_{h}: \mathbb{Z}^{2} \rightarrow \mathbb{Z}$ be the function whose generating function is determined by multiplying $F_{S}(x, y)$ time the first $h$ monomials of $P(x, y)$. Let

$$
Q_{h}(S)=\left\{(i, j) \in \mathbb{Z}^{2}: g_{h}(i, j) \neq 0\right\}
$$

and

$$
\mathcal{P}_{h}=\left\{(i, j) \in Q_{h}(S): g_{h}(i, j)>0\right\}, \quad \mathcal{N}_{h}=\left\{(i, j) \in Q_{h}(S): g_{h}(i, j)<0\right\} .
$$

In particular $Q_{0}(S)=Q(S), \mathcal{P}_{0}=\mathcal{P}$, and $\mathcal{N}_{0}=\mathcal{N}$. Let $M_{h}$ denote the set of multiple points of $Q_{h}(S)$, and let $M_{h}^{+}, M_{h}^{-}$be the subsets of $M_{h}$ formed by the points of $M_{h}$ belonging to $\mathcal{P}_{h}$ and $\mathcal{N}_{h}$, respectively. In particular $M_{0}=\{w\}$, namely the multiple point of $Q(S)$. Moreover, for any pair of sets $X, Y$, we define

$$
X-Y=\{ \pm(u-v), u \in X, v \in Y\} .
$$

Proposition 1. Let $S=\left\{u_{1}, u_{2}, u_{3}, u_{1}+u_{2} \pm u_{3}\right\}$ be a set of four lattice directions. Let $g: \mathbb{Z}^{2} \rightarrow \mathbb{Z}$ be a non trivial function whose generating function is defined by $G_{g}(x, y)=\sum_{t=1}^{r} \delta(t) x^{i(t)} y^{j(t)} F_{S}(x, y)$, where $\delta(t)= \pm 1$. For each $h \in\{1, \ldots, r-1\}$, consider the monomial $\delta(h+1) x^{i(h+1)} y^{i(h+1)}$. Suppose that $\delta(h+1)=1$ and the following conditions hold

1. If $(c, d) \in M_{h}^{+}$then $(i(h+1), i(h+1))=(c, d)-(e, f)$, with $(e, f) \in \mathcal{N}$

2. If $(c, d) \in M_{h}^{-}$then $(i(h+1), i(h+1))=(c, d)-(e, f)$, with $(e, f) \in \mathcal{P}$ 
3. $(i(h+1), i(h+1)) \notin\left(\mathcal{P}_{h}-\mathcal{P}\right) \cup\left(\mathcal{N}_{h}-\mathcal{N}\right)$.

4. $(i(h+1), i(h+1))+w \in \mathcal{N}_{h}$ if $w \in M_{0}^{+},(i(h+1), i(h+1))+w \in \mathcal{P}_{h}$ if $w \in M_{0}^{-}$

Then $M_{h+1} \subseteq M_{h}$

Proof. We give the proof just in the case when both $w$ and $(c, d)$ are positive. The other cases can be obtained by changing the argument in obvious ways. If $(c, d) \in M_{h}^{+}$we add a monomial $x^{i(h+1)} y^{i(h+1)}$ with $(i(h+1), i(h+1))=$ $(c, d)-(e, f),(e, f) \in \mathcal{N}$ and $(i(h+1), i(h+1)) \notin\left(\mathcal{P}_{h}-\mathcal{P}\right) \cup\left(\mathcal{N}_{h}-\mathcal{N}\right)$. This ensures that the negative point $(e, f)$ overlaps $(c, d)$, and consequently that the multiplicity of $(c, d)$ is lowered. Moreover, in order to avoid new multiple points in the updated configuration, we need $(i(h+1), i(h+1)) \notin\left(\mathcal{P}_{h}-\mathcal{P}\right) \cup\left(\mathcal{N}_{h}-\mathcal{N}\right)$, and $(i(h+1), i(h+1))+w \in \mathcal{N}_{h}$.

Remark 2. By the above proposition we can construct a $\{-1,0,+1\}$ valued function $g: \mathbb{Z}^{2} \rightarrow \mathbb{Z}$, with required norm $|g| \leq 1$, starting from a given polynomial $P(x, y) F_{S}(x, y)$ which associated configuration has multiple points. To this we add progressively new monomials according to Proposition 11. See the following example, first part. Of course, we can also work with monomials with negative sign, up to exchange $\mathcal{P}$ with $\mathcal{N}$, and $\mathcal{P}_{h}$ with $\mathcal{N}_{h}$ in the above conditions. See for instance the second part in the following example.

Example 1. Consider the following set of four directions

$$
S=\{(1,0),(0,1),(1,2),(2,1)\}
$$

and the polynomial associated to $S$ :

$$
F_{S}(x, y)=\sum f(i, j) x^{i} y^{j}=(x-1) \cdot(y-1) \cdot\left(x^{2} \cdot y-1\right) \cdot\left(x \cdot y^{2}-1\right)
$$

Let $g_{h}: \mathbb{Z}^{2} \rightarrow \mathbb{Z}$ with generating function given by

$$
\begin{aligned}
& G_{g_{h}}(x, y)=\left(x \cdot y+x^{2} y^{2}+x+x^{2} y\right) \cdot(x-1) \cdot(y-1) \cdot\left(x^{2} \cdot y-1\right) \cdot\left(x \cdot y^{2}-1\right)= \\
& x^{6} \cdot y^{6}-x^{6} \cdot y^{4}-x^{5} \cdot y^{6}+x^{5} \cdot y^{5}-x^{5} \cdot y^{3}+x^{5} \cdot y^{2}-2 \cdot x^{4} \cdot y^{5}+x^{4} \cdot y^{4}+x^{4} \cdot y^{3} \\
& -x^{4} \cdot y^{2}+x^{4} \cdot y+x^{3} \cdot y^{5}-x^{3} \cdot y^{4}+x^{3} \cdot y^{3}+x^{3} \cdot y^{2}-2 \cdot x^{3} \cdot y+x^{2} \cdot y^{4}-x^{2} \cdot y^{3}+ \\
& x^{2} \cdot y-x^{2}-x \cdot y^{2}+x
\end{aligned}
$$

Notice that the multiple negative point $(4,5)$ can be mapped to a simple point by adding the monomial $x^{3} y^{4}$, which corresponds to the choice $(e, f)=(1,1)$. This satisfies the condition $(3,4) \notin\left(\mathcal{P}_{h}-\mathcal{P}\right) \cup\left(\mathcal{N}_{h}-\mathcal{N}\right)$ (since $(i, j)+(3,4) \notin \mathcal{P}_{h}$ for each $(i, j) \in \mathcal{P}$, and $(i, j)+(3,4) \notin \mathcal{N}_{h}$ for each $\left.(i, j) \in \mathcal{N}\right)$. Moreover $(3,4)+(2,2)=(5,6) \in \mathcal{N}_{h}$. Therefore, all conditions in Proposition 1 are fulfilled, and consequently the addition of $x^{3} y^{4}$ does not introduce new multiple points in the updated configuration. Indeed we get 


$$
\begin{aligned}
& G_{g_{h+1}}(x, y)=\left(x \cdot y+x^{2} y^{2}+x+x^{2} y+x^{3} y^{4}\right) \cdot(x-1) \cdot(y-1) \cdot\left(x^{2} \cdot y-1\right) \cdot\left(x \cdot y^{2}-1\right) \\
& =x^{7} \cdot y^{8}-x^{7} \cdot y^{7}-x^{6} \cdot y^{8}+x^{6} \cdot y^{7}+x^{6} \cdot y^{5}-x^{6} \cdot y^{4}-x^{5} \cdot y^{7}+x^{5} \cdot y^{6}-x^{5} \cdot y^{3} \\
& +x^{5} \cdot y^{2}+x^{4} \cdot y^{7}-x^{4} \cdot y^{6}-x^{4} \cdot y^{5}+x^{4} \cdot y^{3}-x^{4} \cdot y^{2}+x^{4} \cdot y+x^{3} \cdot y^{3}+x^{3} \cdot y^{2} \\
& -2 \cdot x^{3} \cdot y+x^{2} \cdot y^{4}-x^{2} \cdot y^{3}+x^{2} \cdot y-x^{2}-x \cdot y^{2}+x
\end{aligned}
$$

so that the number of multiple points decreases.

The multiple point which remains can be eliminated by adding the monomial $x^{3} y$, which corresponds to the choice $(e, f)=(0,0)$. In fact, we have $(i, j)+$ $(3,1) \notin\left(\mathcal{P}_{h+1}-\mathcal{P}\right) \cup\left(\mathcal{N}_{h+1}-\mathcal{N}\right)$, and $(3,1)+(2,2)=(5,3) \in \mathcal{N}_{h+1}$ as before. Therefore, since no new multiple point appears, we get

$$
\begin{aligned}
& \left(x \cdot y+x^{2} y^{2}+x+x^{2} y+x^{3} y^{4}+x^{3} y\right) \cdot(x-1) \cdot(y-1) \cdot\left(x^{2} \cdot y-1\right) \cdot\left(x \cdot y^{2}-1\right)= \\
& x^{7} \cdot y^{8}-x^{7} \cdot y^{7}+x^{7} \cdot y^{5}-x^{7} \cdot y^{4}-x^{6} \cdot y^{8}+x^{6} \cdot y^{7}-x^{6} \cdot y^{3}+x^{6} \cdot y^{2}-x^{5} \cdot y^{7}+x^{5} \cdot y^{6} \\
& -x^{5} \cdot y^{4}+x^{5} \cdot y^{3}+x^{4} \cdot y^{7}-x^{4} \cdot y^{6}-x^{4} \cdot y^{5}+x^{4} \cdot y^{4}+x^{3} \cdot y^{3}-x^{3} \cdot y+x^{2} \cdot y^{4}-x^{2} \cdot y^{3} \\
& +x^{2} \cdot y-x^{2}-x \cdot y^{2}+x
\end{aligned}
$$

which provides a function $g: \mathbb{Z}^{2} \rightarrow \mathbb{Z}$ with $|g| \leq 1$, as required.

We can also add monomials with negative coefficients, by exchanging $\mathcal{P}$ with $\mathcal{N}$, and $\mathcal{P}_{h}$ for $\mathcal{N}_{h}$ in the above conditions. For example, if we start with the polynomial $G_{g_{h}}(x, y)=\left(x \cdot y+x^{2} \cdot y^{2}+x+x^{2} \cdot y\right) \cdot(x-1) \cdot(y-1) \cdot\left(x^{2} \cdot y-1\right) \cdot\left(x \cdot y^{2}-1\right)$ we can continue in the following way.

To eliminate the multiple negative point $-2 x^{4} y^{5}$ we can subtract the monomial $x^{4} y^{4}$, which corresponds to the choice $(e, f)=(1,0) \in \mathcal{N}$, consistent with the conditions $(i, j)+(4,4) \notin \mathcal{N}_{h}$ for each $(i, j) \in \mathcal{P}$, and $(i, j)+(4,4) \notin \mathcal{P}_{h}$ for each $(i, j) \in \mathcal{N}$. Thus we get

$$
\begin{aligned}
& \left(x \cdot y+x^{2} \cdot y^{2}+x+x^{2} \cdot y-x^{4} \cdot y^{4}\right) \cdot(x-1) \cdot(y-1) \cdot\left(x^{2} \cdot y-1\right) \cdot\left(x \cdot y^{2}-1\right) \\
& =-x^{8} \cdot y^{8}+x^{8} \cdot y^{7}+x^{7} \cdot y^{8}-x^{7} \cdot y^{7}+x^{7} \cdot y^{6}-x^{7} \cdot y^{5}+x^{6} \cdot y^{7}-x^{6} \cdot y^{6}+x^{6} \cdot y^{5}- \\
& x^{6} \cdot y^{4}-x^{5} \cdot y^{7}+x^{5} \cdot y^{4}-x^{5} \cdot y^{3}+x^{5} \cdot y^{2}-x^{4} \cdot y^{5}+x^{4} \cdot y^{3}-x^{4} \cdot y^{2}+x^{4} \cdot y+x^{3} \cdot y^{5} \\
& -x^{3} \cdot y^{4}+x^{3} \cdot y^{3}+x^{3} \cdot y^{2}-2 \cdot x^{3} \cdot y+x^{2} \cdot y^{4}-x^{2} \cdot y^{3}+x^{2} \cdot y-x^{2}-x \cdot y^{2}+x
\end{aligned}
$$

The remaining multiple point can be eliminated by subtracting the monomial $x^{3}$, which corresponds to the choice $(e, f)=(0,1) \in \mathcal{N}$, consistent with the conditions $(i, j)+(3,0) \notin \mathcal{N}_{h+1}$ for each $(i, j) \in \mathcal{P}$, and $(i, j)+(4,4) \notin \mathcal{P}_{h+1}$ for each $(i, j) \in \mathcal{N}$. Thus we get

$$
\begin{aligned}
& \left(x \cdot y+x^{2} \cdot y^{2}+x+x^{2} \cdot y-x^{4} \cdot y^{4}-x^{3}\right) \cdot(x-1) \cdot(y-1) \cdot\left(x^{2} \cdot y-1\right) \cdot\left(x \cdot y^{2}-1\right)= \\
& -x^{8} \cdot y^{8}+x^{8} \cdot y^{7}+x^{7} \cdot y^{8}-x^{7} \cdot y^{7}+x^{7} \cdot y^{6}-x^{7} \cdot y^{5}-x^{7} \cdot y^{4}+x^{7} \cdot y^{3}+x^{6} \cdot y^{7}- \\
& x^{6} \cdot y^{6}+x^{6} \cdot y^{5}-x^{6} \cdot y^{3}+x^{6} \cdot y^{2}-x^{6} \cdot y-x^{5} \cdot y^{7}+x^{5} \cdot y^{4}-x^{5} \cdot y^{2}+x^{5} \cdot y-x^{4} \cdot y^{5}+ \\
& x^{4}+x^{3} \cdot y^{5}-x^{3} \cdot y^{4}+x^{3} \cdot y^{3}+x^{3} \cdot y^{2}-x^{3} \cdot y-x^{3}+x^{2} \cdot y^{4}-x^{2} \cdot y^{3}+x^{2} \cdot y-x^{2} \\
& -x \cdot y^{2}+x
\end{aligned}
$$

which provides the generating function of a function $g: \mathbb{Z}^{2} \rightarrow \mathbb{Z}$ with $|g| \leq 1$, as required.

The previous results unable us to deduce the following uniqueness result. 
Corollary 1. $S=\left\{u_{1}, u_{2}, u_{3}, u_{1}+u_{2} \pm u_{3}\right\}$ be a set of four lattice directions. Let $h, g: \mathbb{Z}^{2} \rightarrow\{0,1\}$ be tomographically equivalent with respect to $S$. If

$$
G_{h-g}(x, y)=\sum_{t=1}^{r} \delta(t) x^{i(t)} y^{j(t)} F_{S}(x, y),
$$

with $\delta(t)= \pm 1$, and the degrees $i(t), j(t)$ do not satisfy the conditions of Theorem [3, then $h=g$.

Proof. For the function $\phi=h-g$ it results $|\phi| \leq 1$. Since $G_{\phi}(x, y)$ does not satisfy Theorem 3 then $\phi=0$, and consequently $h=g$.

\section{Conclusion and Perspectives}

In this paper we studied $\{-1,0,+1\}$ valued functions under certain sets of four lattice directions. From their algebraic properties we deduced a uniqueness result. A further step could consist in considering a grid $A$ of fixed size as in the result in 2] in order to show that in fact there exist whole families of suitable valid sets of four directions depending only on the size of $A$, such that any two subsets of $A$ can be distinguished by means of such sets of directions.

\section{References}

1. Hajdu, L., Tijdeman, R.: Algebraic aspects of discrete tomography. J. Reine Angew. Math. 534, 119-128 (2001)

2. Hajdu, L.: Unique reconstruction of bounded sets in discrete tomography. Electron. Notes Discrete Math. 20, 15-25 (2005)

3. Kuba, A., Herman, G.T.: Discrete tomography. Appl. Numer. Harmon. Anal. Birkhäuser, Boston (1999)

4. Kuba, A., Herman, G.T.: Advances in Discrete Tomography and Its Applications. Appl. Numer. Harmon. Anal. Birkhäuser, Boston (2007) 